\title{
Application of contrast media in post-mortem imaging (CT and MRI)
}

\author{
Silke Grabherr $^{1} \cdot$ Jochen Grimm $^{1,2} \cdot$ Pia Baumann $^{1} \cdot$ Patrice Mangin $^{1}$
}

Received: 26 January 2015 / Accepted: 12 March 2015 / Published online: 5 April 2015

(C) Italian Society of Medical Radiology 2015

\begin{abstract}
The application of contrast media in postmortem radiology differs from clinical approaches in living patients. Post-mortem changes in the vascular system and the absence of blood flow lead to specific problems that have to be considered for the performance of post-mortem angiography. In addition, interpreting the images is challenging due to technique-related and post-mortem artefacts that have to be known and that are specific for each applied technique. Although the idea of injecting contrast media is old, classic methods are not simply transferable to modern radiological techniques in forensic medicine, as they are mostly dedicated to single-organ studies or applicable only shortly after death. With the introduction of modern imaging techniques, such as post-mortem computed tomography (PMCT) and post-mortem magnetic resonance (PMMR), to forensic death investigations, intensive research started to explore their advantages and limitations compared to conventional autopsy. PMCT has already become a routine investigation in several centres, and different techniques have been developed to better visualise the vascular system and organ parenchyma in PMCT. In contrast, the use of PMMR is still limited due to practical issues, and research is now starting in the field of PMMR angiography. This article gives an overview of the problems in post-mortem contrast media application, the various classic and modern
\end{abstract}

Silke Grabherr

Silke.Grabherr@chuv.ch

1 University Center of Legal Medicine Lausanne-Geneva, University Hospital of Lausanne, Chemin de la Vulliette 4, 1000 Lausanne 25, Switzerland

2 Department of Diagnostic and Interventional Radiology, University Hospital of Lausanne, Rue du Bugnon 46, 1011 Lausanne, Switzerland techniques, and the issues to consider by using different media.

Keywords Post-mortem computed tomography · Postmortem magnetic resonance imaging · Post-mortem angiography $\cdot$ Contrast media $\cdot$ Virtual autopsy $\cdot$ Forensic radiology

\section{Introduction}

Visualising the vascular system has always been a challenge in medicine. Simple dissection techniques on cadavers were not sufficient for understanding the complex anatomy of this system. Thus, the idea was born to inject substances into the hollow vessels and produce so-called "vascular casts" [1, 2]. At that time, vascular injection was only a preliminary method prior to dissection and preparation of the organ to be investigated. The injected vessels were easier to identify, expose, and visualise. With the discovery of X-rays by Röntgen in 1896, new possibilities for investigating the vascular system arose. Only 3 years later, in 1899, radio-opaque materials were injected into the coronary arteries of isolated human hearts [1]. At that time, the technique of post-mortem angiography (PMA), the injection of radio-opaque contrast media into the vascular system of organs or bodies, was born.

During the last two decades, the research field of postmortem radiology has grown considerably. The application of modern cross-sectional imaging techniques for forensic death investigations has been introduced in medico-legal centres all over the world, especially for multidetector computed tomography (MDCT) and occasionally magnetic resonance imaging (MRI). Since the first report of postmortem computed tomography (PMCT) in 1983 [3], the 
number of examinations has increased significantly [4-13]. Today, PMCT is a routine investigation in some centres. The advantages of PMCT and post-mortem MRI (PMMR) are evident, as they represent a possibility of documenting the interior of a body and serving as proof in medico-legal investigations [14]. Though PMCT represents the best way to visualise the skeletal system $[11,15]$ and MRI being an excellent tool for investigating organ parenchyma and soft tissue [16], none of these techniques are ideally suited for vascular diagnosis. Therefore, the application of contrast media as used in clinical radiology was a promising next step. This development mirrors clinical practice, in which CT-angiography (CTA) is the most widely used method for the detection and localisation of clinically active haemorrhages of unknown origin [17]. CTA is also an excellent method for assessing acute and chronic coronary artery disease [18], as it allows investigation of the arterial lumen and vascular wall diseases.

As most of the classic methods of PMA are only applicable to isolated organs, they were not fit to be reintroduced into modern post-mortem imaging. Also, techniques usually used in clinical angiography are not simply transferable to post-mortem applications, as no vascular circulation is present and hydrosoluble contrast media diffuses rapidly out of the vascular lumen of the deceased. Therefore, the development of specific techniques is necessary to successfully apply contrast media in post-mortem imaging. This article gives an overview of PMA, the existing types of contrast agents, and the modern PMA techniques available today.

\section{Classic PMA methods}

PMA boomed during the first half of the twentieth century. A number of different techniques for PMA existed, and new injection materials and methods were developed so rapidly that it is difficult to follow the development in detail. The range of mixtures reported in Schoenmackers' remarkable publication from 1964 [1] still shows the immense knowledge scientists of that time had about vascular injections. Unfortunately, most of this knowledge has been lost, and from the hundreds of techniques that have been used, only a few are still used in the twenty first century.

As recently proposed [2], the techniques can be divided into six groups according to the nature of the injected material: (1) vascular casts, (2) corpuscular preparations in gelatine or agar, (3) corpuscular preparations in aqueous solution, (4) hydrosoluble preparations, (5) oily liquids, and (6) miscellaneous. As mentioned before, vascular casts represent the oldest method of PMA. Various casting materials have been used over the years. Corpuscular preparations consist of a corpuscular radiopaque material, which is usually soluble in water, and they can be mixed with gelatine or agar, allowing hardening after cooling, a technique originally used for histological sections. Hydrosoluble contrast agents were only rarely used in classic PMA, nearly exclusively for visualising the vascular system of recently deceased foetuses and newborns [19]. Oily liquids were mostly used for perfusion studies, as they remain mostly intravascular, a fact that had already been recognised in 1968 by Barmayer, who perfused coronary arteries with a mixture of diesel and paraffin oil to measure their flow capacity [20]. Similar examples are the use of lipophilic contrast agents, such as Lipiodol Ultra Fluide (iodised oil, Guerbet) [21]. At the zenith of classic PMA (end of nineteenth and beginning of twentieth century), hundreds of injection materials were used to render the vascular system visible for either macroscopic or radiological techniques, mostly for a combination of both. Anatomists and other researchers have created their own injection mixtures, some of them still preserved in Schoenmackers' paper [1], but most of them are probably lost forever.

Although the classic techniques are not the ones applied today, there are many things that we can learn from them in combination with modern post-mortem imaging. Many scientists using the classic techniques were specialists in perfusion of the vascular system of human bodies. These specialists were perfectly aware that the vascular system needs special treatment after death, and that clinical contrast agents are not ideal for post-mortem use. Therefore, they were also experts in the development of perfusion liquids and mixtures of contrast agents. To develop techniques, new contrast agents, new perfusion liquids, and devices for modern PMA, the information contained in the classic literature is essential to avoid unnecessary trials and to choose the best basic material and approach.

\section{Contrast media}

To perform PMA, the simple injection of contrast agent as done in clinical radiology is not sufficient. Due to the cessation of intravascular circulation, haemostasis, agonal procedures, and post-mortem changes, most of the vascular system is empty after death, sometimes partly filled with post-mortem blood clots or gas [22]. To fill this empty system, a high volume of perfusion liquid is necessary [23]. However, adequate perfusion of a human body is complex, and specific perfusion liquids are needed. Every liquid injected into the vascular system has a specific behaviour in the vessels (Fig. 1). This behaviour depends on the kind of liquid (oily, corpuscular mixture, aqueous, etc.) and its physical properties (viscosity, osmolarity, radio-opacity, etc.). With increasing post-mortem delay, the permeability of the vascular wall increases, making it necessary to find a specific contrast agent that produces good vascular contrast without too many 


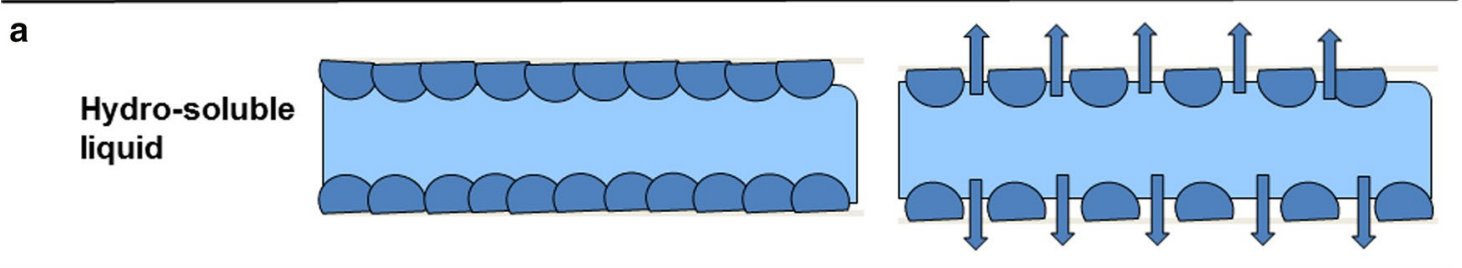

b

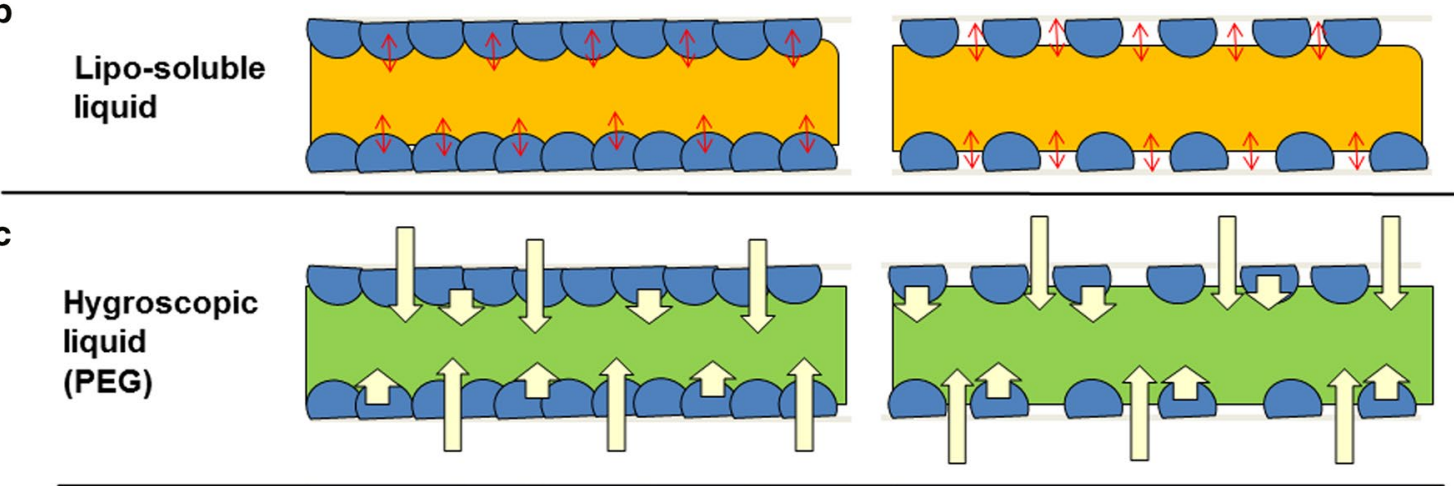

Fig. 1 Demonstration of the behaviour of different liquids in the vascular system. a Due to post-mortem changes, the vascular wall becomes porous, especially due to changes in the endothelium, leading to leakage of hydrosoluble liquids throughout the vessel wall. b Oily liquids remain intravascular because they do not mix with the aqueous milieu. The vessel wall creates a vascular/oily barrier.

artefacts. Thus, clinical aqueous contrast agents may produce sufficient quality directly after death, but they are not suited for PMA at higher post-mortem intervals.

Fuelled by the introduction of MDCT into post-mortem investigations and the goal to enhance PMCT with contrast agent, new research aims to combine PMA with PMCT into post-mortem CT-angiography (PMCTA). The greatest challenge in these research projects was to find an adequate liquid for filling and contrasting the vascular lumen. Two different approaches have been used: one using aqueous liquids and one using oily liquids.

\section{Aqueous liquids}

Although widely used in clinical radiology, hydrosoluble contrast agents face challenges in PMA. Due to changes in the vascular system after death, the permeability of the vascular wall increases significantly. Therefore, any injection of a hydrosoluble liquid leads to rapid extravasation from the vascular lumen into the surrounding tissue [24] (Fig. 1). This effect is well known, and even exploited, as in embalming procedures $[25,26]$ where the increase of turgescence of the soft tissue indicates successful embalming. However, for PMA, the injected contrast agent has to remain intravascular
Hygroscopic liquids can be created after mixing a water-soluble contrast agent with a substance such as polyethylene glycol (PEG). Because they are hyperosmolar, hygroscopic liquids remain intravascular but tend to extract water from the surrounding tissue, leading to desiccation and clumping of the remaining blood

for better vascular contrast and to minimise oedema in the surrounding tissue [24]. Therefore, the application of hydrosoluble liquids remains restricted to a short post-mortem delay. The first application of a simple hydrosoluble contrast agent for PMA was reported in 1866 [27]. In the twentieth century, various water-soluble contrast agents were tested. Although the easy injection and availability of aqueous contrast solutions (mostly expired clinical contrast agents) are an advantage, their use for whole-body PMCTA is limited. Jackowski et al. [28], who injected Telebrix Gastro (ioxithalamate, Guerbet) as a contrast agent, described an important limitation of its use. The aqueous contrast agent solution caused tissue oedema and artefacts in histological investigations, which rendered its application in medico-legal cases difficult [29]. Today, their use in PMA is limited to specific techniques, such as targeted angiography of the coronary arteries [30,31], in which extravasation plays a less important role because only one organ is perfused, or directly after death for PMA using cardiac massage $[32,33]$.

\section{Aqueous liquids mixed with polyethylene glycol (hyperosmolar mixture)}

To limit extravascular diffusion of aqueous liquids, Jackowski et al. [29] proposed adding hydroscopic polyethylene 
glycol (PEG) as a contrast agent dissolver. PEG consists of large polymerised molecules that remain within the vessel lumen. Only a few studies with small numbers of human and animal corpses or organs have shown the possible use of such a contrast agent mixture [29, 34, 35]. Ross et al. [35] compared this mixture to an oily mixture in 10 human cadavers and concluded that the PEG solution was advantageous due to tissue enhancement similar to clinical radiology. He also recognised that the aqueous mixture produces less extravasation in the pancreatic and gastric mucosa than the oily mixture, though the authors used low-viscosity oil, which has never been applied in another study. No literature was found concerning the effect of this mixture on the quality of autopsies carried out after PMCTA and on histology. However, our own experiences have shown some limitations, as the hydroscopic abilities of the mixture tend to attract water from the surrounding tissue (Fig. 1), leading to clumping of the remaining blood in the vessels, which could mask a thrombosis. Also, the contrast agent mixing with blood can be a problem, as quantifying the volume of a vital haemorrhage after PMCTA is no longer possible.

\section{Oily contrast agent mixture}

The fact that oily liquids mostly remain intravascular and occlude microcapillaries limits extravasation into the surrounding tissue and renders them useful for PMA. This was recognised in 1968 by Barmayer [20]. Lack of extravasation and the microembolisation properties of oily agents are also used in clinical radiology, e.g. Lipiodol ${ }^{\circledR}$ [36]. By injecting oily liquids into a body, a post-mortem circulation can be established [24, 37], mimicking in vivo conditions. This can be useful for training medical staff or testing new medical devices [38]. By using perfusion with warm paraffin oil, percutaneous catheterisation can be performed and as an example, a newly developed self-expanding aortic valve can be delivered to the correct place [39]. Oily liquids were introduced to modern forensic radiology by Grabherr and colleagues. The concept of these experiments was that PMCTA should resemble clinical CTA as closely as possible. An initial feasibility study performed on an animal model showed the potential of the concept with the use of diesel oil as a perfusate and a roller pump as the perfusion device [24]. This technique was later adapted to a human model by changing the perfusion liquid and the injection technique [37]. One of the most important parameters for the injection of oily liquids is the viscosity of the oil. This was already described by Schoenmackers et al. [1], who provided a list of different high- and low-viscosity oils that can be used as basic substances for injection mixtures. The viscosity of the oil is a critical determinant of the calibre of the vessels that can be penetrated. With increasing viscosity, the diameter of the microvessels being embolised increases. Visualisation of the mircrovascular system is possible using low-viscosity oil as in micro-angiography [40]. To perform PMA of the human body, a specific viscosity is necessary. If the viscosity is too low, artefactual extravasation is observed, especially in regions of so-called locus minoris resistentiae [24], meaning regions with early bacterial decomposition and autolytic activities, such as the gastro-intestinal tract. If the viscosity is too high, microvascular structures and organ parenchyma lack optimal contrast. Therefore, the specifications of the contrast agent mixture are very important. The Angiofil ${ }^{\circledR} /$ paraffin oil mixture used for multiphase PMCTA (MPMCTA) is an example of a good working mixture [23].

Regardless of the contrast media or perfusate that is injected, they have consequences, as they can potentially alter the composition of fluid-based biological samples and interfere with specific post-mortem investigations. In medico-legal and forensic cases, the analysis of biological fluids is important to define the cause of death or perform toxicological analyses. Prior to the injection of contrast media, it should be determined, whether samples should be taken before the injection. These considerations depend on the injected material and the type of angiography performed (whole-body angiography or targeted angiography). In addition, water-soluble contrast agents will mix with the remaining blood, precluding the possibility of separating the two liquids during autopsy and the extravasated blood cannot be quantified. This is different for oily liquids, which automatically separate from the hydrosoluble blood, enabling quantification during autopsy. Only a few studies have investigated the influence of different contrast media. The available studies pertain exclusively to MPMCTA and TCA and their influence on toxicology [41, 42], biochemistry [43], microbiology [44], genetics [45], histology, and fatty embolism evaluation [46, 47]. Therefore, the recommendation remains that as many specimens as possible should be sampled prior to injection of the contrast medium, as described by Schneider et al. [48].

\section{Modern PMCTA techniques}

As mentioned above, PMCT is clearly the most widespread imaging tool in modern post-mortem radiology. This may be due to the fact that CT scanners are widely available, data acquisition is rapid, and the maintenance costs are affordable for some institutes of legal medicine. The role of PMCT as an adjunct to or replacement for autopsy is discussed controversially in the medico-legal community. Several studies have been performed with the aim of investigating the diagnostic value of PMCT compared to conventional autopsy $[11,13,15]$. PMCT has even been proposed 
Fig. 2 Whole-body PMCTA allows visualization of the vascular system of the head, thorax, and abdomen and two- and three-dimensional reconstructions

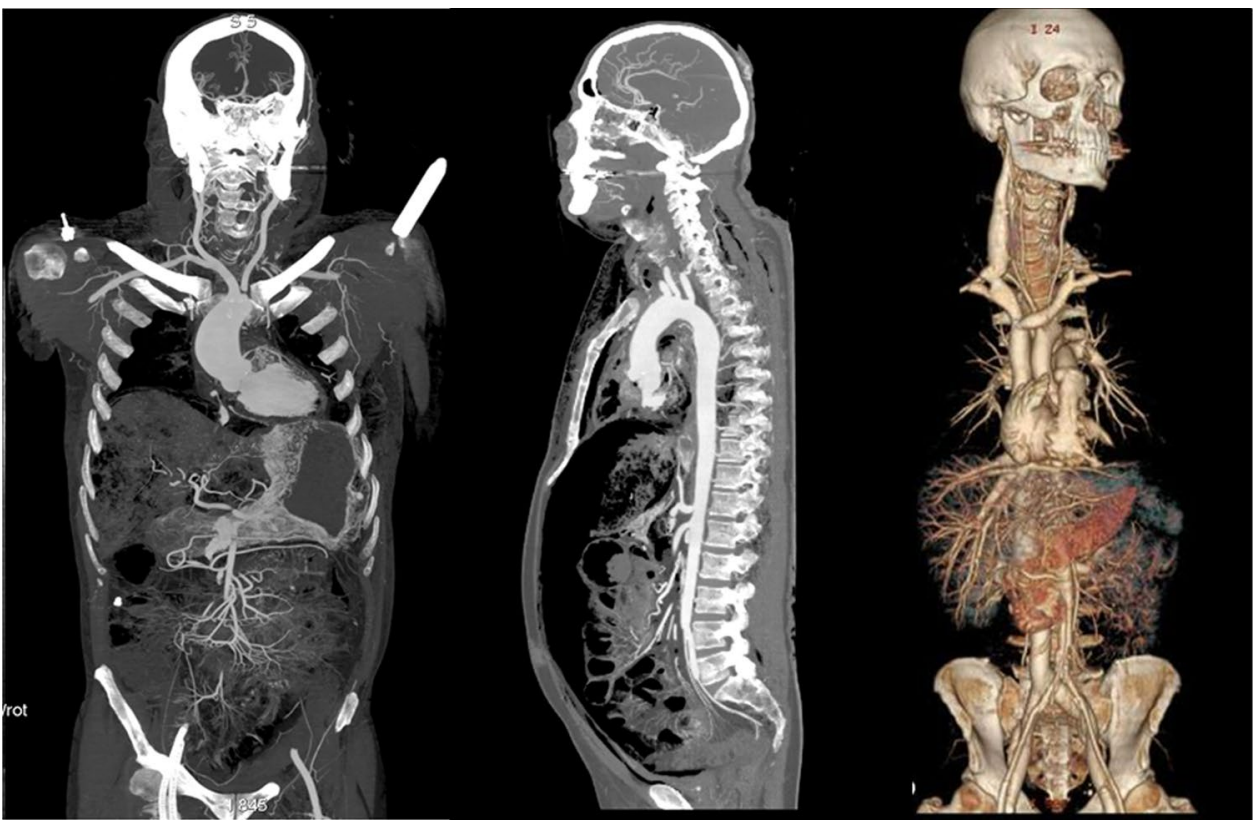

to replace conventional autopsy in certain cases [49, 50]. However, the most important weakness of PMCT is generally accepted to be its limited soft tissue contrast and low ability to visualise the vascular system. The introduction of PMCTA may influence the result of this discussion as it allows detailed visualisation of the vascular system (Fig. 2).

Several research groups across the world have approached PMCTA in different ways, leading to the various methods described in the literature [51]. Though some of the techniques are single experiences [52], others have been applied more regularly.

An overview of the methods is given in Table 1. In general, whole-body PMCTA and targeted coronary PMCTA have to be differentiated.

\section{Whole body PMCTA techniques}

\section{Multiphase post-mortem CT-angiography (MPMCTA)}

The MPMCTA is currently the most used and explored PMCTA technique. Because it was developed specifically for forensic applications, it uses standardised material and injection protocol, which should increase acceptance in court by reaching a complete filling of the vascular system while minimising artefacts, enabling improved radiological interpretation. The method was first published in 2011 [23]. A mixture of medium-viscosity paraffin oil (paraffinum liquidum) and an oily contrast agent (Angiofil ${ }^{\circledR}, 6 \%$ ) is injected via unilateral cannulation of the femoral vessels, or in some cases, the axillar vessels [53]. The injection is performed using a Virtangio ${ }^{\circledR}$ perfusion device. MPMCTA follows a specific injection protocol, standardising the flow rate and injected volume for each angiographic phase (Table 2): an arterial phase showing the arterial system, a venous phase for investigating the venous system, and the final dynamic phase during which PMCT data is acquired simultaneously with perfusion of the body, displaying the vessels during active luminal flow similar to clinical in vivo angiography.

Interpretation of the obtained images needs training and experience, as some post-mortem changes can mimic pathological findings $[54,55]$. However, a recent study showed that the artefacts observed after MPMCTA are reproducible and stable in terms of their localisation and type, making them relatively easy to recognise [56].

A recently published study compared the overall performance of MPMCTA with conventional autopsy and unenhanced PMCT in 50 cases [15]. The results indicate that MPMCTA and conventional autopsy draw similar conclusions regarding causes of death. Though the majority of findings were detected both with autopsy and MPMCTA, the latter demonstrated a higher sensitivity for identifying skeletal and vascular lesions, whereas conventional autopsy provided more information about organ morphology and pathology. Even though MPMCTA discovered slightly fewer lesions than autopsy in general, it detected significantly better the essential lesions. The combination of MPMCTA and autopsy performed best. Therefore, MPMCTA is an extremely powerful and useful tool for post-mortem investigations.

Chevallier et al. [15] included all cases investigated in a medico-legal centre without any inclusion criteria concerning the circumstances and cause of death, but other studies 


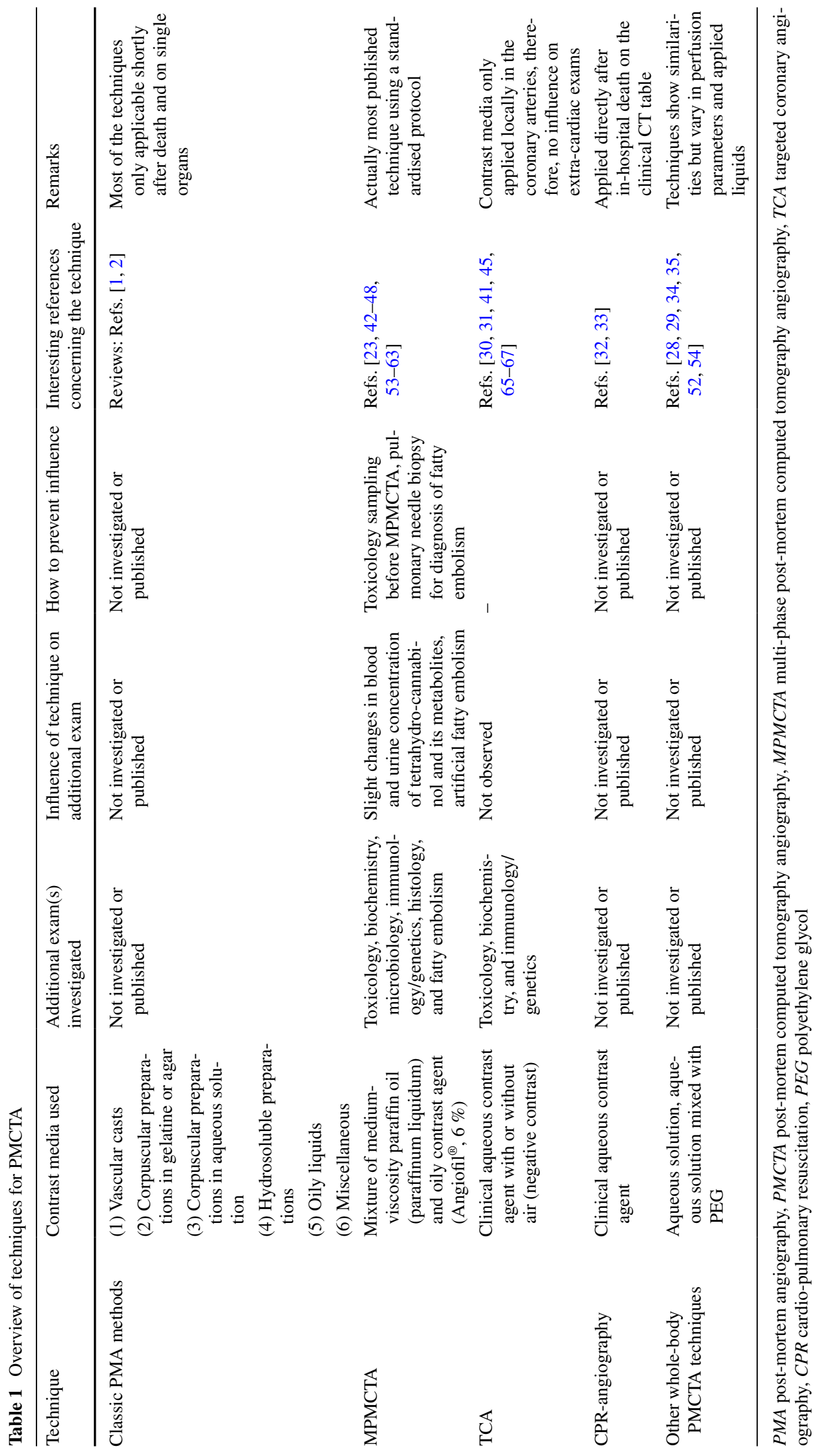


Table 2 Protocol for MPMCTA

\begin{tabular}{lllll}
\hline Angiographic phase & Volume $(\mathrm{ml})$ & Flow rate $(\mathrm{ml} / \mathrm{min})$ & What is visualized/enhanced & Aim \\
\hline Unenhanced MDCT - & - & Skeletal system, large vessels, organs & $\begin{array}{c}\text { Traumatic (bone) lesions, major lesions of } \\
\text { organs/vessels, calcifications of vessels, } \\
\text { determining indication for PMCTA }\end{array}$ \\
Arterial phase & 1200 & 800 & Arterial system (including pulmonary \\
veins) & $\begin{array}{c}\text { Arterial stenosis, occlusions, extravasa- } \\
\text { tions, signs of cardiac ischaemia (patho- } \\
\text { logical enhancement) }\end{array}$ \\
Venous phase & 1800 & 800 & $\begin{array}{c}\text { Arterial and venous system (including } \\
\text { pulmonary arteries), organ parenchyma }\end{array}$ & $\begin{array}{c}\text { Occlusions, venous extravasation, signs of } \\
\text { pulmonary embolism, lesions of organ } \\
\text { parenchyma }\end{array}$ \\
Dynamic phase & 500 & 200 & $\begin{array}{c}\text { Arterial and venous system, organ } \\
\text { parenchyma }\end{array}$ & $\begin{array}{c}\text { Verifying findings from the first two phases } \\
\text { (persistence of filling defects, stenosis, } \\
\text { etc.) }\end{array}$ \\
\hline
\end{tabular}

have been carried out investigating different case groups for which CTA would be indicated in a clinical setting: traumatic cases, in which a source of bleeding should be detected [57], cases of death following surgical intervention [58], and cases of cardiovascular disease [59]. These studies revealed that MPMCTA has considerable diagnostic potential in the detection of sources of bleeding, even more than clinical CTA in living patients [57]. Visualisation of bleeding sources and extravasation of contrast agent can also be used as a tool to visualise the trajectories of projectiles in the case of lethal gun shots, or stab wounds in cases of sharp trauma $[60,61]$. In cases of sudden cardiac death due to ischaemic heart disease, MPMCTA can guide direct sampling for histological examination by identifying stenosis and allow assessment of the morphology of coronary arteries, including stenoses and occlusions [59]. Furthermore, MPMCTA also permits the identification of coronary thrombosis [46]. The high sensitivity and diagnostic potential of MPMCTA makes this technique an effective tool in forensic pathology, but it also shows clear advantages in a clinical pathology setting. A study performed on hospitalised patients who died unexpectedly or within $48 \mathrm{~h}$ of an event necessitating cardiopulmonary resuscitation showed promising results [62], leading the authors to conclude that, in cases of unexpected death, the addition of MPMCTA significantly increases the value of the post-mortem radiological exam, making it a feasible alternative for quality control and the identification of diagnoses traditionally made by medical autopsy.

\section{PMCTA using cardiopulmonary resuscitation (CPR)}

This technique for visualising the vascular system was developed in Japan, where the rate of PMCT is high (over 20,000 cases per year) [12] and, due to traditional reasons, the autopsy rate is very low. For the same traditional reasons, and because any surgical intervention such as the cannulation of vessels in a deceased patient is shunned, an even less invasive and simple technique ideal for hospital application was developed based on the principles of clinical contrast agent injection and CPR [32, 33]. As stated in the literature, CPR can increase blood pressure to a certain degree and generate a small cardiac output [63] due to chest compression. CPR is used to propel the contrast agent through the vascular system. Normal clinical aqueous contrast media is injected into peripheral veins, such as the cubital vein, as in clinical in vivo contrast-enhanced CT investigations. The contrast agent then enters the heart cavities, from where it is dispersed into the pulmonary and body circulation by repeated chest compression. Because this chest compression can cause injuries, such as rib fractures, the technique is preferably performed in cases of prior unsuccessful CPR. The procedure is carried out directly on the CT table for approximately $2 \mathrm{~min}$, followed by a CT scan. The obtained images show contrast agent in large parts of the arterial and venous system depending on the investigated case. Lesions, such as organ injuries and localisation of the source of haemorrhage, are visible due to extravasation of the contrast media. Therefore, the most common indication is currently the investigation of traumatic death in which CPR in the emergency room did not lead to success.

\section{Other methods of whole-body PMCTA}

Some other approaches of whole-body PMCTA have been reported in the literature without indicating specific protocols [28, 29, 34, 35, 52, 54]. One method reported by the Swiss Virtopsy ${ }^{\circledR}$ (http://www.virtopsy.com) team uses an approach similar to MPMCTA; it consists of cannulation of the femoral vessels of one side of the body and the injection of an aqueous contrast agent solution. This method was first described by Jackowski et al. [27] in 2005 and later modified by the authors [29, 34, 35]. Other injection 
protocols using mixtures of aqueous solution with or without PEG have been proposed in the literature, most of them including an arterial and venous phase. Some authors have also proposed leaving the injected contrast agent in the vessels for 15-20 min before performing the CT scan, allowing it to diffuse out of the vascular bed and leading to contrast enhancement [35]. The body was also proposed to be scanned once in a prone position to reach complete filling of the right coronary artery [35]. However, some artefacts were encountered that could not be fully explained (e.g. over-enhancement of neck muscles after failed resuscitation). Unfortunately, the number of cases reported in the literature seems too small to fully appreciate the advantages and disadvantages of the method.

\section{Targeted coronary PMCTA}

In contrast to whole-body PMA techniques, targeted PMCTA techniques $[30,31]$ consist of the filling of a specific part of the vascular system, most commonly, the coronary arteries. This aim can be reached, for example, by the introduction of a urinary catheter designated for bladder catheterisation into the ascending aorta. Regular clinical contrast agent is injected into the aorta manually or with a clinical contrast-injector. As the inflated urinary catheter avoids backflow into the descending aorta, contrast agent is pressed into the coronary arteries. Targeted coronary PMCTA techniques have been developed in England, where the investigation of cardiovascular death has become a major focus [64]. This may be the reason as to why two English centres independently decided to introduce PMCTA of the coronary arteries [30, 31]. Though both techniques use the left carotid artery for access, they have some differences; the method developed in Leicester consists of the injection of both air (negative contrast) and contrast media [65], whereas the one developed in Oxford uses only positive contrast media and makes efforts to avoid air inside the vessels [31]. The injection technique also exhibits variations. The Oxford system uses manual injection into the carotid artery, whereas the research team in Leicester introduced a standard clinical CT power-contrast injector [66], which allows acquisition of CT images of the coronary arteries during ongoing contrast injection, allowing their visualisation similar to the dynamic phase of MPMCTA. Using this approach, a more meaningful assessment of vessel stenosis [67] can be obtained. Because the methods use aqueous contrast agents, rapid extravasation out of the vascular lumen is observed and scanning has to be done during or immediately after injection. However, due to the small quantity of contrast agent applied for targeted PMCTA, no important tissue oedema is observed [66].
A detailed study investigating the performance of TCA, compared these techniques to autopsy and histological investigations of coronary arteries [67], revealing that, similar to whole body approaches, TCA has good results identifying coronary lesions and is suited for investigating ischaemic heart disease. The authors also discuss whether the minimally invasive approach of PMCTA has advantages over the conventional method of investigating coronary vessels, as the invasive autopsy and histology approach represent trauma for the vessels, leading to more important artefacts.

\section{First experiences with PMMR angiography}

Although PMMR is much less frequently used than PMCT, it is a powerful tool with a wide scope in forensic imaging [16]. Due to superior soft tissue contrast, PMMR allows visualisation of anatomical components that are not visible by PMCT, specifically organ parenchyma. Therefore, this technique may represent the missing link for obtaining a minimally invasive autopsy. Reasons for the rare use of PMMR are mostly practical and technical. High costs of imaging equipment, especially high maintenance costs, discourage legal medicine centres from obtaining own MRI units. Data acquisition also requires well-trained specialists not easily available in a medicolegal centre. They could use the equipment of a neighbouring radiological department, but access to a clinical MRI unit for post-mortem imaging may be complicated. PMMR exams are usually performed when the hospital is closed, and this limits the application in daily routine, especially as PMMR is a time-consuming exam compared to PMCT.

Experiences with PMMR are scarce in the literature. Most of the studies have focussed on cases of blunt trauma $[68,69]$ or natural, especially cardiovascular, death [69, 70]. Reading the images is complicated due to artefacts (e.g. MRI contrast is temperature-dependent $[16,71]$ ) and other post-mortem changes. Therefore, differentiation between normal findings and true pathology is difficult [16, 72]. Due to these challenges, research regarding PMMR is much less advanced than for PMCT. Concerning PMMR angiography, only single trials exist. In 2012, Ruder et al. [73] reported whole-body PMMR angiography using clinical aqueous iodinated contrast medium (Optiray $300^{\circledR}$ ) diluted in a solution of PEG. Good image contrast was obtained using fat-saturated T1-weighted images. However, problems were observed due to position-dependent sedimentation of contrast medium that decreased the image quality. This was explained by the long scan time and the absence of circulation of the contrast agent during image acquisition. 
Active research is ongoing to solve this problem, such as that within the Technical Working Group Post-mortem Angiography Methods (TWGPAM). This international research group consisting of different worldwide institutions aims to further develop PMA methods and deliver standardised methods, guidelines, and training for accurate interpretation of the images [55]. In a first step, a protocol has been proposed, allowing the standardised data acquisition of ex situ hearts [74]. At the same time, the possibility of using the contrast agent mixture in the MPMCTA technique (paraffin oil and Angiofil $^{\circledR}$ ) was investigated. The results of this work show that this mixture is an effective contrast agent for PMMR and does not suffer from the artefacts described by Ruder et al. [73]. Therefore, MPMCTA can be combined with PMMRA angiography, which would be especially interesting in cases of cardiovascular disease. As Jackowski [75-77] has shown in several papers, PMMR seems to have the potential to detect cardiac infarction in a way that is more sensitive than conventional autopsy and histology. Although this research has to be further developed, a combination of MPMCTA that allows the detection of coronary stenosis or occlusions with local cardiac PMMR angiography would likely open new possibilities in investigating cardiac death.

\section{Conclusion}

The application of contrast media in post-mortem radiology is a complex subject and differs in many ways from clinical contrast-enhanced radiology. No circulation is present and the permeability of the vascular system is increased after death, requiring the development of specific contrast media mixtures and techniques to perfuse the vessels. Moreover, the interpretation of the images differs from the regular clinical approach, and artefacts exist due to postmortem changes depending on the applied method. Knowledge of these issues is important for correctly interpreting the findings. Furthermore, any injection of perfusion liquids may potentially alter the composition of biological liquids and the findings of subsequent autopsy and/or histological analyses. Although different approaches have been used in PMCTA, standardised techniques for which detailed knowledge about potential consequences exists are rare. However, some techniques are already applied as routine exams and the initial institutional guidelines for interpretation of the images exist. In contrast to PMCTA, PMMR angiography is far from routine application. No standardised methods are yet available, but intensive research is ongoing.

Acknowledgments The first author (Silke Grabherr) has had personal academic funding from the Fondation Leenards, Lausanne, Switzerland.
Conflict of interest The authors declare that they have no conflict of interest.

Ethical standards This article does not contain any studies with human participants or animals performed by any of the authors.

\section{References}

1. Schoenmackers J (1960) Technik der postmortalen angiographie MIT berücksichtigung verwandter methoden postmortaler Gefäßdarstellung. Ergeb Allg Pathol Anat 39:53-151

2. Grabherr S, Djonov V, Yen K, Thali MJ, Dirnhofer R (2007) Postmortem angiography: review of former and current methods. AJR 188:832-838

3. Krantz P, Holtas S (1983) Postmortem computed tomography in a diving fatality. J Comput Assist Tomogr 7:132-134

4. Dirnhofer R, Jackowski C, Vock P, Potter K, Thali MJ (2006) VIRTOPSY: minimally invasive, imaging-guided virtual autopsy. Radiographics 26:1305-1333

5. Thali M, Dirnhofer R, Vock P (2009) The virtopsy approach: 3D optical and radiological scanning and reconstruction in forensic medicine. CRC, New York

6. Weustink AC, Hunink MG, van Dijke CF, Renken NS, Krestin GP, Oosterhuis JW (2009) Minimally invasive autopsy: an alternative to conventional autopsy? Radiology 250:897-904

7. Jeffery AJ (2010) The role of computed tomography in adult postmortem examinations: an overview. Diagn Histopathol $16: 546-551$

8. O'Donnell C (2010) An image of sudden death: utility of routine postmortem computed tomography scanning in medico-legal autopsy practice. Diagn Histopathol 16:552-555

9. Poulsen K, Simonsen J (2007) Computed tomography as a routine in connection with medico-legal autopsies. Forensic Sci Int 171:190-197

10. Jacobsen C, Lynnerup N (2010) Craniocerebral trauma-congruence between postmortem computed tomography diagnoses and autopsy results: a 2-year retrospective study. Forensic Sci Int 194:9-14

11. Roberts IS, Benamore RE, Benbow Jackson A, Mallett S, Patankar T, Peebles C, Roobottom C, Traill ZC (2012) Postmortem imaging as an alternative to autopsy in the diagnosis of adult deaths: a validation study. Lancet 379:136-142

12. Okuda T, Shiotani S, Sakamoto N, Kobayashi T (2013) Background and current status of postmortem imaging in Japan: short history of "Autopsy imaging (Ai)". Forensic Sci Int 225:3-8

13. Kasahara S, Makino Y, Hayakawa M, Yajima D, Iti H, Iwase H (2012) Diagnosable and non-diagnosable causes of death by postmortem computed tomography: a review of 339 forensic cases. Leg Med 14:239-245

14. Pomara C, Fineschi V, Scalzo G, Guglielmi G (2009) Virtopsy versus digital autopsy: virtual autopsy. Radiol Med 114(8):1367-1382

15. Chevallier C, Doenz F, Vaucher P, Palmiere C, Dominguez A, Binaghi S, Mangin P, Grabherr S (2013) Postmortem computed tomography angiography vs. conventional autopsy: advantages and inconveniences of each method. Int J Leg Med 127:981-989

16. Ruder TD, Thali MJ, Hatch GM (2014) Essentials of forensic post-mortem MR imaging in adults. $\mathrm{Br} \mathrm{J}$ Radiol 87(1036):20130567

17. Kennedy DW, Laing CJ, Tseng LH, Rosenblum DI, Tamarkin SW (2010) Detection of active gastrointestinal hemorrhage with CT angiography: a 4(1/2)-year retrospective review. J Vasc Interv Radiol 21:848-855 
18. Deo R, Albert CM (2010) Epidemiology and genetics of sudden cardiac death. Circulation 125:620-637

19. Foote GA, Wilson AJ, Steward JH (1978) Perinatal post-mortem radiography: experience with 2500 cases. $\mathrm{Br} \mathrm{J}$ Radiol 51:351-356

20. Barmeyer J (1968) Postmortale koronarangiographie und perfusion normaler und pathologisch veränderter herzen, messung der durchflusskapazität interkoronarer anastomosen. Beitr Pathol Anat 137:373-390

21. Pfeifer KJ, Klein U, Chaussy C, Hammer C, Pielsticker K, Haendle H, Lissner J (1974) Postmortale nierenvergrößerungsangiographie mit fettlöslichem kontrastmittel. Fortschr Röntegenstr 121:472-476

22. Egger C, Bize $P$, Vaucher $P$, Mosimann $P$, Schneider B, Dominguez A, Meuli R, Mangin P, Grabherr S (2012) Distribution of artefactual gas on post-mortem multidetector computed tomography (MDCT). Int J Leg Med 126:3-12

23. Grabherr S, Doenz F, Steger B, Dirnhofer R, Dominguez A, Sollberger B, Gygax E, Rizzo E, Chevallier C, Meuli R, Mangin P (2011) Multi-phase post-mortem CT-angiography development of a standardized protocol. Int J Leg Med 125:791-802

24. Grabherr S, Djonov V, Friess A, Thali MJ, Ranner G, Vock P, Dirnhofer R (2006) Postmortem angiography after vascular perfusion with diesel oil and a lipophilic contrast agent. AJR 187:W515-W523

25. Grabuschnigg P, Rous F (1990) Preservation of human cadavers throughout history: a contribution to development and methodology. Beitr Gerichtl Med 48:455-458

26. Macdonald GJ, Macgregor DB (1998) Procedures for embalming cadavers for the dissecting laboratory. Proc Soc Exp Biol Med 215:363-365

27. Chrzonszczewsky N (1866) Zur anatomie und physiologie der leber. Virchows Arch Path Anat 35:153

28. Jackowski C, Thali M, Sonnenschein M, Aghayev E, von Allmen G, Yen K, Dirnhofer R, Vock P (2005) Virtopsy: postmortem minimally invasive angiography using cross section techniques-implementation and preliminary results. J Forensic Sci 50:1175-1186

29. Jackowski C, Bolliger S, Aghayev E, Christe A, Kilchoer T, Aebi B, Périnat T, Dirnhofer R, Thali MJ (2006) Reduction of postmortem angiography-induced tissue edema by using polyethylene glycol as a contrast agent dissolver. J Forensic Sci 5:1134-1137

30. Saunders SL, Morgan B, Raj V, Robinson CE, Rutty GN (2011) Targeted post-mortem computed tomography cardiac angiography: proof of concept. Int J Leg Med 125:609-616

31. Roberts IS, Benamore RE, Peebles C, Roobottom C, Traill ZC (2011) Diagnosis of coronary artery disease using minimally invasive autopsy: evaluation of a novel method of post-mortem coronary CT angiography. Clin Radiol 66:645-650

32. Sakamoto N, Senoo S, Kamimura Y, Uemura K (2009) Case report: cardiopulmonary arrest on arrival case which underwent contrast-enhanced postmortem CT. J Jpn Assoc Acute Med 30:114-115

33. Lizuka K, Sakamoto N, Kawasaki H, Miyoshi T, Komatsuzaki A, Kikuchi S (2009) Usefulness of contrast-enhanced postmortem CT. Innervision 24:89-92

34. Jackowski C, Persson A, Thali MJ (2008) Whole body postmortem angiography with a high viscosity contrast agent solution using poly ethylene glycol as contrast agent dissolver. J Forensic Sci 53:465-468

35. Ross S, Spendlove D, Bolliger S, Christe A, Oesterhelweg L, Grabherr S, Thali MJ, Gygax E (2008) Postmortem whole-body CT angiography: evaluation of two contrast media solutions. AJR 190:1380-1389

36. Nakakuma $\mathrm{K}$, Tashiro S, Hiraoka $\mathrm{T}$, Uemura $\mathrm{K}$, Konno $\mathrm{T}$, Miyauchi Y, Yokoyama I (1983) Studies on anticancer treatment with an oily anticancer drug injected into the ligated feeding hepatic artery for liver cancer. Cancer 52:2193-2200

37. Grabherr S, Gygax E, Sollberger B, Ross S, Oesterhelweg L, Bolliger S, Christe A, Djonov V, Thali MJ, Dirnhofer R (2008) Two-step post-mortem angiography with a modified heart-lung machine: preliminary results. AJR 190:345-351

38. Willaert W, Van Hoof T, De Somer F, Grabherr S, D'Herde K, Ceelen W, Pattyn P (2014) Postmortem pump-driven reperfusion of the vascular system of porcine lungs: towards a new model for surgical training. Eur Surg Res 52:8-20

39. Chevallier C, Willaert W, Kawa E, Centola M, Steger B, Dirnhofer R, Mangin P, Grabherr S (2014) Postmortem Circulation: a new model for testing endovascular devices and training clinicians in their use. Clin Anat 27:556-562

40. Grabherr S, Hess A, Karolczak M, Thali MJ, Friess S, Kalender W, Irnhofer R, Djonov V (2008) Angiofil ${ }^{\circledR}{ }_{\text {-mediated visualiza- }}$ tion of the vascular system by microcomputed tomography: a feasibility study. MRT 71:551-556

41. Rutty GN, Smith P, Barber J, Amorosa J, Morgan B (2013) The effect on toxicology, biochemistry and immunology investigations by the use of targeted post-mortem computed tomography angiography. Forensic Sci Int 225:42-47

42. Palmiere C, Grabherr S, Augsburger M (2014) Postmortem computed tomography angiography, contrast medium administration and toxicological analyses in urine. Leg Med. http://dx.doi. org/10.1016/j.legalmed.2014.12.005 (epub, ahead of print)

43. Grabherr S, Widmer C, Iglesias K, Sporkert F, Augsburger M, Mangin P, Palmiere C (2012) Postmortem biochemistry performed on vitreous humor after postmortem CT-angiography. Leg Med 14:297-303

44. Palmiere C, Egger C, Grabherr S, Jaton-Ogay K, Greub G (2014) Postmortem angiography using femoral cannulation and postmortem microbiology. Int J Leg Med. doi:10.1007/ s00414-014-1099-5

45. Rutty GN, Barber J, Amoroso J, Morgan B, Graham EA (2013) The effect on cadaver DNA identification by the use of targeted and whole body post-mortem computed tomography angiography. Forensic Sci Med Pathol 9:489-495

46. Palmiere C, Lobrinus JA, Mangin P, Grabherr S (2013) Detection of coronary thrombosis after multi-phase postmortem CTangiography. Leg Med 15:12-18

47. Capuani C, Guilbeau-Frugier C, Mokrane FZ, Delisle MB, Marcheix B, Rousseau H, Telmon N, Rougé D, Dedouit F (2014) Tissue microscopic changes and artefacts in multi-phase postmortem computed tomography angiography in a hospital setting: a fatal case of systemic vasculitis. Forensic Sci Int 242:e12-e17. doi:10.1016/j.forsciint.2014.06.039 (epub, ahead of print)

48. Schneider B, Chevallier C, Dominguez A, Bruguier C, Elandoy C, Mangin P, Grabherr S (2012) The Forensic radiographer: a new member in the medico-legal team. Am J Forensic Med Pathol 33:30-36

49. Donchin Y, Rivkind AI, Barziv J, Hiss J, Almog J, Drescher M (1994) Utility of postmortem computed-tomography in trauma victims. J Trauma Infect Crit Care 37:552-556

50. Wichmann D, Obbelode F, Vogel H, Hoepker WW, Nierhaus A, Braune S, Sauter G, Pueschel K, Kluge S (2012) Virtual autopsy as an alternative to traditional medical autopsy in the intensive care unit: a prospective cohort study. Ann Intern Med 156:123-130

51. Saunders S, Morgan B, Raj V, Rutty G (2010) Post-mortem computed tomography angiography: past, present and future. Forensic Sci Med Pathol 7:271-277

52. Jolibert M, Cohen F, Bartoli C, Boval C, Vidal V, Gaubert JY, Moulin G, Petit P, Bartoli JM, Leonetti G, Gorincour G (2011) Postmortem CT-angiography: feasibility of US-guided vascular access. J Radiol 92:446-449 
53. Pomara C1, Bello S, Grilli G, Guglielmi G, Turillazzi E (2014). Multi-phase postmortem CT angiography (MPMCTA): a new axillary approach suitable in fatal thromboembolism. Radiol Med (epub ahead of print)

54. Berger N, Martinez R, Winklhofer S, Flach PM, Ross S, Ampanozi G, Gascho D, Thali MJ, Ruder TD (2013) Pitfalls in postmortem CT-angiography-intravascular contrast induces postmortem pericardial effusion. Leg Med 15(6):315-317

55. Grabherr S, Wittig H, Dedouit F, Wozniak K, Vogel H, Heinemann A, Fischer F, Moskala A, Guglielmi G, Mangin P, Grimm J (2015) Letter on Pitfalls in post-mortem CT-angiography_-Intravascular contrast induces post-mortem pericardial effusion. Leg Med (epub ahead of print)

56. Bruguier C, Mosimann PJ, Vaucher P, Uské A, Doenz F, Jackowski C, Mangin P, Grabherr S (2013) Multi-phase postmortem $\mathrm{CT}$ angiography: recognizing technique-related artefacts and pitfalls. Int J Leg Med 127:639-652

57. Palmiere C, Binaghi S, Doenz F, Bize P, Chevallier C, Mangin P, Grabherr S (2012) Detection of hemorrhage source: the diagnostic value of post-mortem CT-angiography. Forensic Sci Int 222:33-39

58. Zerlauth JB, Doenz F, Dominguez A, Palmiere C, Uské A, Meuli R, Grabherr S (2013) Surgical interventions with fatal outcome: utility of multi-phase postmortem CT angiography. Forensic Sci Int 225:32-41

59. Michaud K, Grabherr S, Doenz F, Mangin P (2012) Evaluation of postmortem MDCT and MDCT-angiography for the investigation of sudden cardiac death related to atherosclerotic coronary artery disease. Int J Cardiovasc Imaging 28:1807-1822

60. Moskała A, Woźniak K, Kluza P, Bolechała F, RzepeckaWoźniak E, Kołodziej J, Latacz K (2012) Validity of post-mortem computed tomography angiography (PMCTA) in medicolegal diagnostic management of stab and incised wounds. Arch Med Sadowej Kryminol. 62:315-326

61. Grabherr S, Grimm J (2014) Multiphase post-mortem CT-angiography (MPMCTA): a new method for investigation violent death. In: Vogel H (ed) Forensics, radiology, society X-rays: tool and document Dr. Kovač. Verlag, Hamburg

62. Wichmann D, Heinemann A, Weinberg C, Vogel H, Hoepker WW, Grabherr S, Pueschel K, Kluge S (2014) Virtual autopsy with multiphase postmortem computed tomographic angiography versus traditional medical autopsy to investigate unexpected deaths of hospitalized patients: a cohort study. Ann Intern Med 160(8):534-541

63. Jackson RE, Freeman SB (1983) Hemodynamics of cardiac massage. Emerg Med Clin North Am 1:501-513

64. Dorries C (2004) Coroners' courts: a guide to law and practice, 2nd edn. Oxford University Press, Oxford

65. Rutty G, Raj V, Saunders S, Morgan B (2011) Air as a contrast medium for targeted post-mortem computed tomography cardiac angiography. Acad Forensic Pathol 1:144-145
66. Robinson B, Barber J, Morgan B, Rutty GN (2012) Pump injector system applied to targeted post-mortem coronary artery angiography. Int J Leg Med 127:661-666

67. Morgan B, Biggs MJ, Barber J, Raj V, Amoroso J, Hollingbury FE, Robinson C, Rutty GN (2013) Accuracy of targeted postmortem computed tomography coronary angiography compared to assessment of serial histological sections. Int J Leg Med 2013(127):809-817

68. Yen K, Vock P, Tiefenthaler B, Ranner G, Scheurer E, Thali MJ, Zwygart K, Sonnenschein M, Wiltgen M, Dirnhofer R (2004) Virtopsy: forensic traumatology of the subcutaneous fatty tissue; multislice computed tomography (MSCT) and magnetic resonance imaging (MRI) as diagnostic tool. J Forensic Sci 49:799-806

69. Ruder TD, Ebert LC, Khattab AA, Rieben R, Thali MJ (2013) Edema is a sign of early acute myocardial infarction on postmortem magnetic resonance imaging. Forensic Sci Med Pathol. doi:10.1007/s12024-013-9459-x (epub ahead of print)

70. Cha JG, Kim DH, Kim DH, Paik SH, Park JS, Park SJ, Lee HK, Hong HS, Choi DL, Yang KM, Chung NE, Lee BW, Seo JS (2010) Utility of postmortem autopsy via whole-body imaging: initial observations comparing MDCT and 3.0 T MRI findings with autopsy findings. Korean J Radiol 11:395-406

71. Nelson TR, Tung SM (1987) Temperature dependence of proton relaxation times in vitro. Magn Reson Imaging 5:189-199

72. Drew T, Evans K, Võ ML, Jacobson FL, Wolfe JM (2013) Informatics in radiology: what can you see in a single glance and how might this guide visual search in medical images? Radiographics $33: 263-274$

73. Ruder TD, Hatch GM, Ebert LC, Flach PM, Ross S, Ampanozi G, Thali MJ (2012) Whole body postmortem magnetic resonance angiography. J Forensic Sci 57:778-782

74. Bruguier C, Egger C, Vallée JP, Grimm J, Boulanger X, Jackowski C, Mangin P, Grabherr S (2014) Postmortem magnetic resonance imaging of the heart ex situ: development of technical protocols. Int J Leg Med (epub ahead of print)

75. Jackowski C, Warntjes MJ, Berge J, Bär W, Persson A (2011) Magnetic resonance imaging goes postmortem: noninvasive detection and assessment of myocardial infarction by postmortem MRI. Eur Radiol 21:70-78

76. Jackowski C, Schwendener N, Grabherr S, Persson A (2013) Post-mortem cardiac 3-T magnetic resonance imaging: visualization of sudden cardiac death? J Am Coll Cardiol 62:617-629

77. Jackowski C, Hofmann K, Schwendener N, Schweitzer W, Keller-Sutter M (2012) Coronary thrombus and peracute myocardial infarction visualized by unenhanced postmortem MRI prior to autopsy. Forensic Sci Int 214:e16-e19 